\title{
UNIVERSALITY LIMITS AT THE HARD EDGE OF THE SPECTRUM FOR MEASURES WITH COMPACT SUPPORT
}

\author{
D. S. LUBINSKY
}

\begin{abstract}
We use the theory of entire functions and reproducing kernels to establish universality at the (hard) edge of the spectrum for a measure with compact support. This involves the Bessel kernel. In particular, we show that universality at the hard edge is equivalent to universality along the diagonal at the hard edge.
\end{abstract}

\section{RESUlts ${ }^{1}$}

Let $\mu$ be a finite positive Borel measure with compact support $\operatorname{supp}[\mu]$. Then we may define orthonormal polynomials

$$
p_{n}(x)=\gamma_{n} x^{n}+\ldots, \gamma_{n}>0,
$$

$n=0,1,2, \ldots$ satisfying the orthonormality conditions

$$
\int p_{n} p_{m} d \mu=\delta_{m n}
$$

One of the key limits in random matrix theory, the so-called universality limit, involves the reproducing kernel

$$
K_{n}(x, y)=\sum_{k=0}^{n-1} p_{k}(x) p_{k}(y)
$$

and its normalized cousin

$$
\widetilde{K}_{n}(x, y)=\mu^{\prime}(x)^{1 / 2} \mu^{\prime}(y)^{1 / 2} K_{n}(x, y) .
$$

For $x$ in the interior of $\operatorname{supp}[\mu]$ (the "bulk" of the support), at least when $\mu^{\prime}(x)$ is finite and positive, the universality limit typically takes the form

$$
\lim _{n \rightarrow \infty} \frac{\widetilde{K}_{n}\left(x+\frac{a}{\widetilde{K}_{n}(x, x)}, x+\frac{b}{\widetilde{K}_{n}(x, x)}\right)}{\widetilde{K}_{n}(x, x)}=\frac{\sin \pi(a-b)}{\pi(a-b)},
$$

Date: February 26, 2008.

${ }^{1}$ Research supported by NSF grant DMS0400446 and US-Israel BSF grant 2004353 
uniformly for $a, b$ in compact subsets of the real line. There is a large literature on this. Some recent references are [3], [14], [16], [17], [18], [19], [20], [27], [31].

For $x \in \operatorname{supp}[\mu] \backslash(\operatorname{supp}[\mu])^{o}$ (the so-called hard edge of the spectrum), the situation is more complicated, and the most general results involve comparison to Jacobi weights on $(-1,1)$. Until recently, the most general such result is due to Kuijlaars and Vanlessen [14]. Let $\mu$ be absolutely continuous, and $\mu$ have the form

$$
d \mu(x)=h(x) w^{(a, \beta)}(x) d x=h(x)(1-x)^{\alpha}(1+x)^{\beta} d x,
$$

where $h$ is positive and analytic in $[-1,1]$. They showed (together with many other powerful results) that uniformly for $a, b$ in bounded subsets of $(0, \infty)$, as $n \rightarrow \infty$, the limit involves the Bessel kernel of order $\alpha$ :

$$
\frac{1}{2 n^{2}} \tilde{K}_{n}\left(1-\frac{a}{2 n^{2}}, 1-\frac{b}{2 n^{2}}\right)=\mathbb{J}_{\alpha}(a, b)+O\left(\frac{a^{\alpha / 2} b^{\alpha / 2}}{n}\right) .
$$

Here if $u \neq v$,

$$
\mathbb{J}_{\alpha}(u, v)=\frac{J_{\alpha}(\sqrt{u}) \sqrt{v} J_{\alpha}^{\prime}(\sqrt{v})-J_{\alpha}(\sqrt{v}) \sqrt{u} J_{\alpha}^{\prime}(\sqrt{u})}{2(u-v)},
$$

while

$$
\mathbb{J}_{\alpha}(u, u)=\frac{1}{4}\left\{J_{\alpha}^{2}(\sqrt{u})-J_{\alpha+1}(\sqrt{u}) J_{\alpha-1}(\sqrt{u})\right\},
$$

and $J_{\alpha}$ is the usual Bessel function of the first kind and order $\alpha$,

$$
J_{\alpha}(z)=\sum_{n=0}^{\infty} \frac{(-1)^{n}(z / 2)^{2 n+\alpha}}{n ! \Gamma(\alpha+n+1)} .
$$

In [19], we used a comparison method to prove a more general result, for so-called regular measures. We say that $\mu$ is regular on $[-1,1]$ in the sense of Ullmann and/ or Stahl and Totik [28], if

$$
\lim _{n \rightarrow \infty} \gamma_{n}^{1 / n}=2
$$

\section{Theorem 1.1}

Let $\mu$ be a finite positive Borel measure on $(-1,1)$ that is regular. Assume that for some $\rho>0, \mu$ is absolutely continuous in $J=$ $[1-\rho, 1]$, and in $J$, its absolutely continuous component has the form $w=h w^{(\alpha, \beta)}$, where $\alpha, \beta>-1$. Assume that $h(1)>0$ and $h$ is continuous at 1 . Then uniformly for $a, b$ in compact subsets of $(0, \infty)$, we have

$$
\lim _{n \rightarrow \infty} \frac{1}{2 n^{2}} \tilde{K}_{n}\left(1-\frac{a}{2 n^{2}}, 1-\frac{b}{2 n^{2}}\right)=\mathbb{J}_{\alpha}(a, b) .
$$


If $\alpha \geq 0$, we may allow compact subsets of $[0, \infty)$.

The problem with the method of [19] is that it requires a comparison measure with a similar support, for which universality at the edge is known. In that paper, the comparison measure was the classic Jacobi weight.

In this paper, we present a different method, based on the theory of entire functions and reproducing kernels, which removes this restriction. It is the analogue for the hard edge, of a method that has worked well in the bulk, for measures on compact sets [20] and for exponential and varying weights [17]. It should in principle allow an extension of Theorem 1.1 to measures with general compact support.

We let

$$
J_{\alpha}^{*}(z)=J_{\alpha}(z) / z^{\alpha}
$$

and

$$
\mathbb{J}_{\alpha}^{*}(z, v)=\mathbb{J}_{\alpha}(z, v) /\left\{z^{\alpha / 2} v^{\alpha / 2}\right\} .
$$

The advantage of $J_{\alpha}^{*}$ and $\mathbb{J}_{\alpha}^{*}$ over $J_{\alpha}$ and $\mathbb{J}_{\alpha}$ respectively, is that the former are entire. We shall use them to prove a version of the universality limit (1.7) that holds also for $a, b$ at 0 , and more generally, for $a, b$ in compact subsets of the plane.

To motivate the formulation, we examine universality at the hard edge for the Jacobi weight

$$
w^{\alpha, 0}(x)=(1-x)^{\alpha}, x \in(-1,1) .
$$

For this weight, with reproducing kernel $K_{n}^{\alpha, 0}$, we have [21, p. 85], [29, (4.5.8), p. 72],

$$
\lim _{n \rightarrow \infty} K_{n}^{\alpha, 0}(1,1)\left(\frac{1}{2 n^{2}}\right)^{\alpha+1}=\frac{1}{2^{2 \alpha+2} \Gamma(\alpha+1) \Gamma(\alpha+2)}=\mathbb{J}_{\alpha}^{*}(0,0) .
$$

Hence for Jacobi weights,

$$
\left(\frac{\mathbb{J}_{\alpha}^{*}(0,0)}{K_{n}^{\alpha, 0}(1,1)}\right)^{1 /(\alpha+1)}=\frac{1}{2 n^{2}}(1+o(1)),
$$

and if we define

$$
\eta_{n}=\left(\frac{\mathbb{J}_{\alpha}^{*}(0,0)}{K_{n}^{\alpha, 0}(1,1)}\right)^{1 /(\alpha+1)},
$$

and recall that $\tilde{K}_{n}$ is the normalized kernel, then we may reformulate (1.7) for the Jacobi weight as

$$
\lim _{n \rightarrow \infty} \frac{1}{2 n^{2}}\left(\frac{a}{2 n^{2}}\right)^{\alpha / 2}\left(\frac{b}{2 n^{2}}\right)^{\alpha / 2} K_{n}^{\alpha, 0}\left(1-\eta_{n} a, 1-\eta_{n} b\right)=\mathbb{J}_{\alpha}(a, b),
$$


or,

$$
\lim _{n \rightarrow \infty} \frac{K_{n}^{\alpha, 0}\left(1-\eta_{n} a, 1-\eta_{n} b\right)}{K_{n}^{\alpha, 0}(1,1)}=\frac{\mathbb{J}_{\alpha}^{*}(a, b)}{\mathbb{J}_{\alpha}^{*}(0,0)} .
$$

It is this form that we shall generalize:

\section{Theorem 1.2}

Let $\mu$ be a finite positive Borel measure with compact support, such that for some $\varepsilon_{0}>0$,

$$
\operatorname{supp}[\mu] \cap\left(1,1+\varepsilon_{0}\right)=\varnothing .
$$

Assume that $\mu$ is absolutely continuous in $\left[1-\varepsilon_{0}, 1\right]$ and for some $\alpha>-1$,

$$
\lim _{x \rightarrow 1-}(1-x)^{-\alpha} \mu^{\prime}(x)=1
$$

Let

$$
\eta_{n}=\left(\frac{\mathbb{J}_{\alpha}^{*}(0,0)}{K_{n}(1,1)}\right)^{1 /(\alpha+1)}, n \geq 1 .
$$

The following are equivalent:

(I) For each real a,

$$
\lim _{n \rightarrow \infty} \frac{K_{n}\left(1-a^{2} \eta_{n}, 1-a^{2} \eta_{n}\right)}{K_{n}(1,1)}=\frac{\mathbb{J}_{\alpha}^{*}\left(a^{2}, a^{2}\right)}{\mathbb{J}_{\alpha}^{*}(0,0)} .
$$

(II) Uniformly for $a, b$ in compact subsets of the complex plane,

$$
\lim _{n \rightarrow \infty} \frac{K_{n}\left(1-a^{2} \eta_{n}, 1-b^{2} \eta_{n}\right)}{K_{n}(1,1)}=\frac{\mathbb{J}_{\alpha}^{*}\left(a^{2}, b^{2}\right)}{\mathbb{J}_{\alpha}^{*}(0,0)} .
$$

\section{Remarks}

(a) The theorem shows that universality along the diagonal (where $b=a$ ) is equivalent to universality in general. Because of the extremal property

$$
1 / K_{n}(x, x)=\inf _{\operatorname{deg}(P)<n} \int P^{2}(t) d \mu(t) / P^{2}(x)
$$

it is far easier to establish universality along the diagonal. Moreover, asymptotics for the Christoffel function $\lambda_{n}(x)=1 / K_{n}(x, x)$ have been studied for decades and are reasonably well understood [5], [21], [22], [30].

(b) Of course, (1.16) certainly holds when for some constant $c$, and uniformly for $a$ in compact subsets of the real line,

$$
\lim _{n \rightarrow \infty} \frac{K_{n}\left(1-a^{2} \eta_{n}, 1-a^{2} \eta_{n}\right)}{\left(2 n^{2}\right)^{1+\alpha}}=c \mathbb{J}_{\alpha}^{*}\left(a^{2}, a^{2}\right) .
$$


When $\mu$ is a measure regular in the sense of Stahl and Totik on $[-1,1]$, and $\mu^{\prime}$ is absolutely continuous in $\left[1-\varepsilon_{0}, 1\right]$, for some $\varepsilon_{0}>0$, and satisfies (1.14), then this last limit was established in [19] by comparing $\mu$ to a Jacobi weight, for which (1.18) is known.

(c) It is likely that (1.18) holds for measures with compact support consisting of finitely many intervals, and satisfying (1.14) and (1.15) at the right endpoint of one of the intervals of support. However it does not seem to have been formally done in the literature. It is also likely that (1.16) can hold even when (1.18) fails.

(d) We use $a^{2}$ and $b^{2}$ rather than $a$ and $b$, for later convenience.

(e) Of course, with trivial changes to the proof, we can assume, instead of (1.14), the more general limit

$$
\lim _{x \rightarrow 1-}(1-x)^{-\alpha} \mu^{\prime}(x)=c>0 .
$$

The paper is organised as follows. In Section 2, we record our notation, and present some background on orthogonal polynomials, and the theory of entire functions of exponential type. In Section 3, we begin with a Bernstein type growth lemma for the plane (Lemma 3.1), and then proceed to obtain estimates on Christoffel functions (Lemma 3.2). Our main lemma in Section 4 is Lemma 4.1, where the normality of the functions $\left\{f_{n}\right\}$ is established. In Lemmas 4.2 to 4.4 , we establish properties of the subsequential limit $f$, and its zeros. In Section 5, we use Markov-Stieltjes inequalities to study the zero distribution of the entire function $f$, and then prove Theorem 1.2. In Section 6, we establish some properties of the Bessel kernel, especially its reproducing kernel property. These properties are used in Section 5.

\section{Notation AND BACKGRound}

In this section, we record our notation, though some of it has already been introduced earlier. In the sequel $C, C_{1}, C_{2}, \ldots$ denote constants independent of $n, x, y, s, t$. The same symbol does not necessarily denote the same constant in different occurences. We shall write $C=C(\alpha)$ or $C \neq C(\alpha)$ to respectively denote dependence on, or independence of, the parameter $\alpha$. We use $\sim$ in the following sense: given real sequences $\left\{c_{n}\right\},\left\{d_{n}\right\}$, we write

$$
c_{n} \sim d_{n}
$$

if there exist positive constants $C_{1}, C_{2}$ with

$$
C_{1} \leq c_{n} / d_{n} \leq C_{2}
$$

Similar notation is used for functions and sequences of functions. 
Throughout, $\mu$ denotes a finite positive Borel measure with compact support. Its Radon-Nikodym derivative is $w=\mu^{\prime}$. The corresponding orthonormal polynomials are denoted by $\left\{p_{n}\right\}_{n=0}^{\infty}$, so that

$$
\int p_{n} p_{m} d \mu=\delta_{m n}
$$

We denote the zeros of $p_{n}$ by

$$
x_{n n}<x_{n-1, n}<\ldots<x_{2 n}<x_{1 n} .
$$

The reproducing kernel $K_{n}(x, t)$, is defined by (1.1), while the normalized reproducing kernel is defined by (1.2). The $n$th Christoffel function is

$$
\lambda_{n}(x)=1 / K_{n}(x, x)=\inf _{\operatorname{deg}(P) \leq n-1} \frac{\int P^{2} d \mu}{P^{2}(x)} .
$$

More generally, $\lambda_{n}(u, x)$ denotes the $n$th Christoffel function for a weight $u$. The Gauss quadrature formula asserts that whenever $P$ is a polynomial of degree $\leq 2 n-1$,

$$
\sum_{j=1}^{n} \lambda_{n}\left(x_{j n}\right) P\left(x_{j n}\right)=\int P d \mu .
$$

In addition to this, we shall need another Gauss type of quadrature formula [5, p. $19 \mathrm{ff}$.]. There are $n$ or $n-1$ points $t_{j n}=t_{j n}(1)$, one of which is 1 , such that

$$
\sum_{j} \lambda_{n}\left(t_{j n}\right) P\left(t_{j n}\right)=\int P d \mu,
$$

whenever $P$ is a polynomial of degree $\leq 2 n-2$. The $\left\{t_{j n}\right\}$ are zeros of

$$
\psi_{n}(1, t)=p_{n}(1) p_{n-1}(t)-p_{n-1}(1) p_{n}(t) .
$$

Because we wish to focus on the point 1 , we shall set $t_{0 n}=1$, and order the $\left\{t_{j n}\right\}$ around 1 , treated as the origin:

$$
\ldots<t_{2, n}<t_{1, n}<t_{0 n}=1<t_{-1, n}<\ldots .
$$

Of course the sequence $\left\{t_{j n}\right\}$ consists of either $n-1$ or $n$ points, so terminates, and it is possible that all $t_{j n}$ lie to the left of 1 . It is known $\left[5\right.$, p. 19] that if $\left(p_{n} p_{n-1}\right)(1) \neq 0$, then one zero of $\psi_{n}(1, t)$ lies in $\left(x_{j n}, x_{j-1, n}\right)$ for each $j$, and the remaining zero lies outside $\left(x_{n n}, x_{1 n}\right)$. In particular, this is the case when $\operatorname{supp}[\mu] \subset(-\infty, 1]$. If $\left(p_{n} p_{n-1}\right)(1)=0$, then $\psi_{n}(1, t)$ is a multiple of $p_{n}$ or $p_{n-1}$, and then again, one zero lies in $\left[x_{j-1, n}, x_{j n}\right)$ for each $j$.

Throughout, $\varepsilon_{0}>0$ is such that

$$
\operatorname{supp}[\mu] \cap\left(1,1+\varepsilon_{0}\right)=\varnothing .
$$


We also assume that $\mu$ is absolutely continuous in $\left[1-\varepsilon_{0}, 1\right]$ and for some $\alpha>-1$,

$$
\lim _{x \rightarrow 1-}(1-x)^{-\alpha} \mu^{\prime}(x)=1 .
$$

Throughout, $J_{\alpha}$ is the Bessel function of order $\alpha$, defined by (1.6), while $J_{\alpha}^{*}$ is its entire cousin defined by (1.8). Moreover, the Bessel kernel $\mathbb{J}_{\alpha}$ is defined by $(1.4)$ and (1.5), while its entire cousin is $\mathbb{J}_{\alpha}^{*}$, defined by (1.9). We shall define for $n \geq 1$,

$$
\eta_{n}=\left(\frac{\mathbb{J}_{\alpha}^{*}(0,0)}{K_{n}(1,1)}\right)^{1 /(\alpha+1)},
$$

and for all complex $a, b$,

$$
f_{n}(a, b)=\frac{K_{n}\left(1-a^{2} \eta_{n}, 1-b^{2} \eta_{n}\right)}{K_{n}(1,1)} .
$$

The zeros of

$$
f_{n}(0, s)=\frac{K_{n}\left(1,1-s^{2} \eta_{n}\right)}{K_{n}(1,1)}
$$

will be denote by $\left\{\rho_{j n}\right\}_{j \neq 0}$. Since $\left\{t_{j n}\right\}$ are the zeros of $\psi_{n}(1, t)=$ $\left(\frac{\gamma_{n-1}}{\gamma_{n}}\right)^{-1}(1-t) K_{n}(1, t)$, we have for $j \neq 0$,

$$
t_{j n}=1-\eta_{n} \rho_{j n}^{2}
$$

We also set,

$$
\rho_{0 n}=0,
$$

corresponding to $t_{0 n}=1$.

For an appropriate subsequence $\mathcal{S}$ of integers, we shall let

$$
f(a, b)=\lim _{n \rightarrow \infty, n \in \mathcal{S}} f_{n}(a, b) .
$$

The zeros of $f(0, \cdot)$ will be denoted by $\left\{\rho_{j}\right\}_{j \neq 0}$, and we set $\rho_{0}=0$. Our ordering of zeros is

$$
0<\rho_{1} \leq \rho_{2} \leq \ldots
$$

We shall denote the (exponential) type of $f(a, \cdot)$ by $\sigma_{a}$. We shall show that $\sigma_{a}$ is independent of $a$, and then just use $\sigma$ to denote the type. Initially, this type will be associated with the specific subsequence $\mathcal{S}$.

We next review some theory that we shall use about entire functions of exponential type. Most of this can be found in the elegant series of 
lectures of B. Ja. Levin [15]. Recall that if $g$ is entire of order 1, then its exponential type $\sigma$ is

$$
\sigma=\limsup _{r \rightarrow \infty} \frac{\max _{|z|=r} \log |g(z)|}{r} .
$$

We say that an entire function $g$ belongs to the Cartwright class and write $g \in \mathcal{C}$ if it is of exponential type and

$$
\int_{-\infty}^{\infty} \frac{\log ^{+}|g(t)|}{1+t^{2}} d t<\infty
$$

Here $\log ^{+} s=\max \{0, \log s\}$.

We let $n(g, r)$ denote the number of zeros of $g$ in the ball center 0 , radius $r$, counting multiplicity. An important result is that for $g \in \mathcal{C}$, that is real valued on the real axis,

$$
\lim _{r \rightarrow \infty} \frac{n(g, r)}{2 r}=\frac{\sigma}{\pi}
$$

For this, see [15, Theorem 1, p. 127]. A simpler proof, in the case of $g$ having all real zeros, is given in [13, p. 66].

When $g$ is entire of exponential type $\sigma$ and bounded along the real axis, we have $[15$, p. 38, Theorem 3]

$$
|g(z)| \leq e^{\sigma|\operatorname{Im} z|}\|g\|_{L_{\infty}(\mathbb{R})}, z \in \mathbb{C} .
$$

When $g$ is entire of exponential type $\sigma$ and $g \in L_{2}(\mathbb{R})$, we write, as did Levin, $g \in L_{\sigma}^{2}$. Here, we have instead of the last inequality, [15, p. $149]$

$$
|g(z)| \leq\left(\frac{2}{\pi}\right)^{1 / 2} e^{\sigma(|\operatorname{Im} z|+1)}\|g\|_{L_{2}(\mathbb{R})}, z \in \mathbb{C}
$$

\section{Bounds on Polynomials and Christoffel Functions}

Throughout, we assume the hypotheses of Theorem 1.2. We start with a consequence of a Bernstein growth lemma:

\section{Lemma 3.1}

Let $[c, 1]$ be a non-empty interval and $\beta \in \mathbb{R}$. Let $\mathcal{K}$ be a compact subset of $\mathbb{C}$. Then there exists $n_{0}=n_{0}(\mathcal{K})$ such that for $n \geq n_{0}, a \in \mathcal{K}$, and polynomials $P$ of degree $\leq n$ satisfying

$$
|P(x)|\left(1-x+\frac{1}{n^{2}}\right)^{\beta} \leq 1, x \in[c, 1],
$$


we have if $\beta \geq 0$,

$$
\left|P\left(1-\frac{a^{2}}{n^{2}}\right)\right| \leq C_{1}\left|\frac{1+a^{2}}{n^{2}}\right|^{-\beta} e^{C_{2}|\operatorname{Im}(a)|} .
$$

and if $\beta<0$, with the added restriction $\left|1 \pm \sqrt{-a^{2}}\right| \geq 1$,

$$
\left|P\left(1-\frac{a^{2}}{n^{2}}\right)\right| \leq C_{1}\left|\frac{1+\sqrt{-a^{2}}}{n}\right|^{2|\beta|} e^{C_{2}|\operatorname{Im}(a)|} .
$$

Here $C_{j} \neq C_{j}(n, P, a, \mathcal{K})$. In particular, for $|a| \geq 2$, and any $\beta$,

$$
\left|P\left(1-\frac{a^{2}}{n^{2}}\right)\right| \leq C_{1}\left(\frac{|a|}{n}\right)^{-2 \beta} e^{C_{2}|\operatorname{Im}(a)|} .
$$

Proof for the case $\beta \geq 0$

We assume $[c, 1]=[-1,1]$. The general case follows by a linear transformation. We may also assume that $P$ has degree $n$. (If not, consider $P(x)+\varepsilon x^{n}$ and then let $\left.\varepsilon \rightarrow 0+\right)$. Let

$$
G(z)=\log |P(z)|+\beta \log \left|1-z+\frac{1}{n^{2}}\right|-(n+\beta) \log |\varphi(z)|,
$$

$z \in \mathbb{C}$, where

$$
\varphi(z)=z+\sqrt{z^{2}-1} .
$$

Then $G$ is subharmonic in the plane, has a finite limit at $\infty$, and is nonpositive on $[-1,1]$ by (3.1). By the maximum principle for subharmonic functions,

$$
G \leq 0 \text { in } \mathbb{C} .
$$

Now let $a \in \mathbb{C}$ and

$$
z=1-\frac{a^{2}}{n^{2}} .
$$

Then

$$
\log |P(z)| \leq-\beta \log \left|1-z+\frac{1}{n^{2}}\right|+(n+\beta) \log |\varphi(z)|
$$


Here if $a^{2} \notin(-\infty, 0)$,

$$
\begin{aligned}
\log |\varphi(z)| & =\log \left|1-\frac{a^{2}}{n^{2}}+\sqrt{-\frac{2 a^{2}}{n^{2}}+\frac{a^{4}}{n^{4}}}\right| \\
& =\operatorname{Re}\left(\frac{\sqrt{-2 a^{2}}}{n}\right)+O\left(\frac{|a|^{2}}{n^{2}}\right) \\
& =\frac{\sqrt{2}|\operatorname{Im}(a)|}{n}+O\left(\frac{|a|^{2}}{n^{2}}\right) .
\end{aligned}
$$

This latter estimate also holds trivially if $a^{2} \in(-\infty, 0)$. Moreover,

$$
\log \left|1-z+\frac{1}{n^{2}}\right|=\log \left|\frac{1+a^{2}}{n^{2}}\right| .
$$

So, uniformly for $a \in \mathcal{K}$,

$$
\log \left|P\left(1-\frac{a^{2}}{n^{2}}\right)\right| \leq-\beta \log \left|\frac{1+a^{2}}{n^{2}}\right|+\sqrt{2}|\operatorname{Im}(a)|+O\left(\frac{1}{n}\right) .
$$

Then (3.2) follows.

Proof for the case $\beta<0$.

Let $g(z, w)$ denote the Green's function for the domain $\mathbb{C} \backslash[-1,1]$ with pole at $w \notin[-1,1]$. Thus, $g(z, w)+\log |z-w|$ is bounded as $z \rightarrow w$, while $g(z, w)$ is harmonic in $\mathbb{C} \backslash([-1,1] \cup\{w\})$, and has limit 0 as $z$ approaches points in $(-1,1)$. An explicit formula for $g(z, w)$ is

$$
g(z, w)=\log \left|\frac{1-\varphi(z) \overline{\varphi(w)}}{\varphi(z)-\varphi(w)}\right| .
$$

This can easily be derived by combining the explicit formula for the Green's function for the unit ball [24, p. 109], with the simple way in which Green's functions are transformed under conformal maps [24, Theorem 4.4.4, p. 107].

We can take the Green's function $g\left(z, 1+\frac{1}{n^{2}}\right)$ with pole at $1+\frac{1}{n^{2}}$, and let

$G(z)=\log |P(z)|+\beta\left\{\log \left|1-z+\frac{1}{n^{2}}\right|+g\left(z, 1+\frac{1}{n^{2}}\right)\right\}-(n+\beta) \log |\varphi(z)|$.

Here $\log \left|1-z+\frac{1}{n^{2}}\right|+g\left(z, 1+\frac{1}{n^{2}}\right)$ is harmonic outside $[-1,1]$, and grows like $\log |z|+O(1)$ at $\infty$, while $g\left(\cdot, 1+\frac{1}{n^{2}}\right)=0$ on $[-1,1]$, so as above, we obtain

$$
G \leq 0 \text { in } \mathbb{C} \text {. }
$$


Then

$\log |P(z)| \leq-\beta\left\{\log \left|1-z+\frac{1}{n^{2}}\right|+g\left(z, 1+\frac{1}{n^{2}}\right)\right\}+(n+\beta) \log |\varphi(z)|$.

Taking $z=1-\frac{a^{2}}{n^{2}}$, we obtain, as above, uniformly for $a \in \mathcal{K}$,

$\log |P(z)| \leq-\beta\left\{\log \left|\frac{1+a^{2}}{n^{2}}\right|+g\left(1-\frac{a^{2}}{n^{2}}, 1+\frac{1}{n^{2}}\right)\right\}+\sqrt{2}|\operatorname{Im}(a)|+O\left(\frac{1}{n}\right)$

so we need to estimate above $g\left(1-\frac{a^{2}}{n^{2}}, 1+\frac{1}{n^{2}}\right)$. We emphasize that the order term is independent of $n, P, a \in \mathcal{K}$. First,

$$
\begin{gathered}
\varphi\left(1-\frac{a^{2}}{n^{2}}\right)=1+\frac{\sqrt{-2 a^{2}}}{n}-\frac{a^{2}}{n^{2}}+O\left(\frac{1}{n^{3}}\right) ; \\
\varphi\left(1+\frac{1}{n^{2}}\right)=1+\frac{\sqrt{2}}{n}+\frac{1}{n^{2}}+O\left(\frac{1}{n^{3}}\right),
\end{gathered}
$$

so uniformly for $a \in \mathcal{K}$, provided $\left|1-\sqrt{-a^{2}}\right| \geq 1$,

$$
\begin{aligned}
& \left|\varphi\left(1+\frac{1}{n^{2}}\right)-\varphi\left(1-\frac{a^{2}}{n^{2}}\right)\right| \\
= & \frac{\sqrt{2}}{n}\left|1-\sqrt{-a^{2}}\right|\left|1+\frac{1+\sqrt{-a^{2}}}{\sqrt{2} n}+O\left(\frac{1}{n^{2}}\right)\right| .
\end{aligned}
$$

Then

$$
\begin{aligned}
& \log \left|\varphi\left(1-\frac{a^{2}}{n^{2}}\right)-\varphi\left(1+\frac{1}{n^{2}}\right)\right| \\
= & \log \left(\frac{\sqrt{2}}{n}\left|1-\sqrt{-a^{2}}\right|\right)+\operatorname{Re}\left(\frac{1+\sqrt{-a^{2}}}{\sqrt{2} n}\right)+O\left(\frac{1}{n^{2}}\right),
\end{aligned}
$$

provided $\left|1-\sqrt{-a^{2}}\right| \geq 1$. Next, from (3.6), uniformly for $a \in \mathcal{K}$,

$$
\begin{aligned}
& 1-\varphi\left(1-\frac{a^{2}}{n^{2}}\right) \overline{\varphi\left(1+\frac{1}{n^{2}}\right)} \\
= & -\frac{\sqrt{2}}{n}\left(1+\sqrt{-a^{2}}\right)-\left(\frac{1+\sqrt{-a^{2}}}{n}\right)^{2}+O\left(\frac{1}{n^{3}}\right) .
\end{aligned}
$$


Then provided $\left|1+\sqrt{-a^{2}}\right| \geq 1$,

$$
\begin{aligned}
& \log \left|1-\varphi\left(1-\frac{a^{2}}{n^{2}}\right) \overline{\varphi\left(1+\frac{1}{n^{2}}\right)}\right| \\
= & \log \left(\frac{\sqrt{2}}{n}\left|1+\sqrt{-a^{2}}\right|\right)+\operatorname{Re}\left(\frac{1+\sqrt{-a^{2}}}{\sqrt{2} n}\right)+O\left(\frac{1}{n^{2}}\right),
\end{aligned}
$$

SO

$$
\begin{aligned}
g(z, w) & =\log \left|1-\varphi\left(1-\frac{a^{2}}{n^{2}}\right) \overline{\varphi\left(1+\frac{1}{n^{2}}\right)}\right|-\log \left|\varphi\left(1-\frac{a^{2}}{n^{2}}\right)-\varphi\left(1+\frac{1}{n^{2}}\right)\right| \\
& =\log \left|\frac{1+\sqrt{-a^{2}}}{1-\sqrt{-a^{2}}}\right|+O\left(\frac{1}{n^{2}}\right) .
\end{aligned}
$$

Then

$$
\begin{aligned}
& -\beta\left\{\log \left|\frac{1+a^{2}}{n^{2}}\right|+g\left(1-\frac{a^{2}}{n^{2}}, 1+\frac{1}{n^{2}}\right)\right\} \\
= & 2|\beta| \log \left|\frac{1+\sqrt{-a^{2}}}{n}\right|+O\left(\frac{1}{n}\right) .
\end{aligned}
$$

So uniformly for $a \in \mathcal{K}$, with $\left|1 \pm \sqrt{-a^{2}}\right| \geq 1$, (3.5) gives

$$
\log \left|P\left(1-\frac{a^{2}}{n^{2}}\right)\right| \leq 2|\beta| \log \left|\frac{1+\sqrt{-a^{2}}}{n}\right|+\sqrt{2}|\operatorname{Im}(a)|+O\left(\frac{1}{n}\right),
$$

and hence (3.3) follows. In particular, (3.4) follows if $|a| \geq 2$.

\section{Lemma 3.2}

There exists $\varepsilon_{1}>0$ such that for $n \geq 1$ and $x \in\left[1-\varepsilon_{1}, 1\right]$,

$$
\lambda_{n}(x) \sim \frac{1}{n}\left(1-x+\frac{1}{n^{2}}\right)^{\alpha+\frac{1}{2}}
$$

Moreover,

$$
\eta_{n}=\left(\frac{\mathbb{J}_{\alpha}^{*}(0,0)}{K_{n}(1,1)}\right)^{1 /(\alpha+1)} \sim n^{-2}
$$

\section{Proof}

We prove this by comparing $\mu$ above and below to suitable measures.

\section{The lower bound}

Let $\varepsilon_{1} \in(0,1)$ and

$$
w_{1}(x)=(1-x)^{\alpha}, x \in\left[1-\varepsilon_{1}, 1\right],
$$


and $w_{1}=0$ elsewhere. By our hypothesis (2.8), for appropriate $\varepsilon_{1}>$ 0 ,we have

$$
\mu^{\prime} \geq \frac{1}{2} w_{1} \text { in }\left[1-\varepsilon_{1}, 1\right]
$$

and hence

$$
\lambda_{n}(x) \geq \frac{1}{2} \lambda_{n}\left(w_{1}, x\right) \text { for } x \in\left[1-\varepsilon_{1}, 1\right] .
$$

Let $L$ denote the linear transformation of $\left[1-\varepsilon_{1}, 1\right]$ onto $[-1,1]$, so that

Also, let

$$
L(t)=-1+\frac{2}{\varepsilon_{1}}\left[t-\left(1-\varepsilon_{1}\right)\right] .
$$

$$
w^{\alpha, 0}(x)=(1-x)^{\alpha}, x \in[-1,1] .
$$

Then

$$
w^{\alpha, 0}(L(t))=\left(\frac{2}{\varepsilon_{1}}\right)^{\alpha} w_{1}(t), t \in\left[1-\varepsilon_{1}, 1\right]
$$

and a simple substitution shows that

$$
\lambda_{n}\left(w_{1}, x\right)=\left(\frac{\varepsilon_{1}}{2}\right)^{\alpha+1} \lambda_{n}\left(w^{\alpha, 0}, L(x)\right) .
$$

Standard estimates $\left[21\right.$, Theorem 28, p. 120] for $\lambda_{n}\left(w^{\alpha}, L(x)\right)$ give for $x \in\left[1-\varepsilon_{1}, 1\right]$,

$$
\lambda_{n}\left(w_{1}, x\right) \sim \frac{1}{n}\left(1-L(x)+\frac{1}{n^{2}}\right)^{\alpha+\frac{1}{2}} \sim \frac{1}{n}\left(1-x+\frac{1}{n^{2}}\right)^{\alpha+\frac{1}{2}},
$$

and hence

$$
\lambda_{n}(x) \geq \frac{C}{n}\left(1-x+\frac{1}{n^{2}}\right)^{\alpha+\frac{1}{2}} .
$$

\section{The Upper Bound}

Choose $a<1<c<d$ such that

$$
\operatorname{supp}[\mu] \subset[a, 1] \cup[c, d]=: \mathcal{K} .
$$

Let

$$
L_{1}(t)=1-\delta(t-1)(t-c) \text {, }
$$

where $\delta$ is a small positive number, so small that

$$
L(t) \in[0,1] \text { for all } t \in \mathcal{K} .
$$

Observe that

$$
1-L(t) \sim 1-t, t \in[a, 1]
$$

and

$$
L^{\prime}(t)=2 \delta\left(\frac{1+c}{2}-t\right) \sim 1 \text { for } t \in[a, 1]
$$


Let $K_{n}^{\alpha, 0}$ denote the kernel corresponding to $w^{\alpha, 0}$. We have for all $x$,

$$
\begin{aligned}
& \int_{1-\varepsilon_{1}}^{1} K_{[n / 2]}^{\alpha, 0}(L(t), L(x))^{2}(1-t)^{\alpha} d t \\
\leq & C \int_{1-\varepsilon_{1}}^{1} K_{[n / 2]}^{\alpha, 0}(L(t), L(x))^{2}(1-L(t))^{\alpha} L^{\prime}(t) d t \\
(3.10= & C \int_{-1}^{1} K_{[n / 2]}^{\alpha, 0}(s, L(x))^{2}(1-s)^{\alpha} d s=C K_{[n / 2]}^{\alpha, 0}(L(x), L(x)) .
\end{aligned}
$$

Note too $\left[21\right.$, Theorem 28, p. 120] that for some $C_{0}>0, n \geq 2$,

$$
\left|K_{[n / 2]}^{\alpha, 0}(u, v)\right| \leq n^{C_{0}}, u, v \in[-1,1] .
$$

Now fix $x \in\left[1-\varepsilon_{1}, 1\right]$ and choose a fast decreasing polynomial $R$ of degree $\leq n / 2-1$ such that $R(x)=1$, while

$$
|R| \leq 1 \text { in }[a, d]
$$

and for some $C>0$,

$$
|R(t)| \leq e^{-C n}, t \in[a, d] \text { with }|t-1| \geq \varepsilon_{1}
$$

Such polynomials were constructed by Ivanov and Totik [12], [25, p. 313]. Let

$$
P(t)=R(t) K_{[n / 2]}^{\alpha, 0}(L(t), L(x))
$$

a polynomial of degree $\leq n-1$, with

$$
P(x)=K_{[n / 2]}^{\alpha, 0}(L(x), L(x)) \sim n\left(1-L(x)+\frac{1}{n^{2}}\right)^{-\left(\alpha+\frac{1}{2}\right)} \sim n\left(1-x+\frac{1}{n^{2}}\right)^{-\left(\alpha+\frac{1}{2}\right)} .
$$

Then using (3.10), (3.11), (3.12),

$$
\begin{aligned}
\lambda_{n}(x) \leq & \int P^{2}(t) d \mu(t) / P^{2}(x) \\
= & \left(\int_{1-\varepsilon_{1}}^{1}+\int_{\operatorname{supp}[\mu] \backslash\left[1-\varepsilon_{1}, 1\right]}\right) P^{2} d \mu / P^{2}(x) \\
\leq & C \int_{1-\varepsilon_{1}}^{1} K_{[n / 2]}^{\alpha, 0}(L(t), L(x))^{2}(1-t)^{\alpha} d t / K_{[n / 2]}^{\alpha, 0}(L(x), L(x))^{2} \\
& +e^{-2 C n} n^{2 C_{0}} \int_{\operatorname{supp}[\mu] \backslash\left[1-\varepsilon_{1}, 1\right]} d \mu / / K_{[n / 2]}^{\alpha, 0}(L(x), L(x))^{2} \\
\leq & C / K_{[n / 2]}^{\alpha, 0}(L(x), L(x))+C e^{-2 C n} n^{2 C_{0}} \\
\leq & \frac{C}{n}\left(1-x+\frac{1}{n^{2}}\right)^{\alpha+\frac{1}{2}} .
\end{aligned}
$$


Together, this and (3.9) give (3.7), and hence (3.8).

\section{Normality}

Recall that

$$
f_{n}(a, b)=\frac{K_{n}\left(1-a^{2} \eta_{n}, 1-b^{2} \eta_{n}\right)}{K_{n}(1,1)}
$$

where $\eta_{n}$ satisfies (3.8).

\section{Lemma 4.1}

(a) $\left\{f_{n}(a, b)\right\}$ is uniformly bounded for $a, b$ in compact subsets of the plane.

(b) If $f(\cdot, \cdot)$ is the uniform limit of some subsequence $\left\{f_{n}\right\}_{n \in \mathcal{S}}$, then $f$ is entire and even in $a, b$, and

$$
|f(a, b)| \leq C_{1}(1+|a|)^{-(2 \alpha+1)}(1+|b|)^{-(2 \alpha+1)} e^{C_{2}[|\operatorname{Im}(a)|+|\operatorname{Im}(b)|]} .
$$

Here $C_{j} \neq C_{j}(a, b), j=1,2$. In particular, $f(a, \cdot)$ is entire of exponential type and bounded on the real axis.

(c) For each real $a, f(a, \cdot)$ has at most two non-real zeros, consisting of at most one pair of conjugate zeros on the imaginary axis.

Proof

(a) By Cauchy-Schwarz and the previous lemmas, we have

$$
\begin{aligned}
& \frac{1}{n}\left|\left(1-x+\frac{1}{n^{2}}\right)^{\frac{\alpha+\frac{1}{2}}{2}}\left(1-t+\frac{1}{n^{2}}\right)^{\frac{\alpha+\frac{1}{2}}{2}} K_{n}(x, t)\right| \\
\leq & \left(\frac{1}{n}\left(1-x+\frac{1}{n^{2}}\right)^{\alpha+\frac{1}{2}} K_{n}(x, x)\right)^{1 / 2}\left(\frac{1}{n}\left(1-t+\frac{1}{n^{2}}\right)^{\alpha+\frac{1}{2}} K_{n}(t, t)\right)^{1 / 2} \leq C
\end{aligned}
$$

for $x, t \in\left[1-\varepsilon_{1}, 1\right]$. Recall too that

$$
K_{n}(1,1) \sim n^{2 \alpha+2} \text { and } \eta_{n} \sim n^{-2} .
$$

Let $A>2$. By (3.4) of Lemma 3.1, applied separately in each variable, we then have for $2 \leq|a|,|b| \leq A$, and $n \geq n_{0}(A)$,

$$
\begin{array}{r}
\left|f_{n}(a, b)\right|=\left|\frac{K_{n}\left(1-a^{2} \eta_{n}, 1-b^{2} \eta_{n}\right)}{K_{n}(1,1)}\right| \\
\leq C_{1}|a|^{-\left(\alpha+\frac{1}{2}\right)}|b|^{-\left(\alpha+\frac{1}{2}\right)} e^{C_{2}[|\operatorname{Im}(a)|+|\operatorname{Im}(b)|]} .
\end{array}
$$

Here $C_{1}$ and $C_{2}$ are independent of $a, b$. Since $f_{n}$ is analytic in each variable, the maximum-modulus principle also yields a uniform bound for $|a|,|b| \leq 2$. This establishes the uniform boundedness of $\left\{f_{n}(\cdot, \cdot)\right\}$. (b) Since $\left\{f_{n}\right\}$ is a sequence of polynomials, the bound in (a) shows that $\left\{f_{n}(a, b)\right\}$ is a normal family for $a, b$ in compact subsets of $\mathbb{C}$. Then if $f$ is the limit of the subsequence $\left\{f_{n}\right\}_{n \in \mathcal{S}}$, the bound (4.2) follows 
for $2 \leq|a|,|b| \leq A$, and by the maximum-modulus principle also for $|a|,|b| \leq 2$. Moreover, $f$ is even in $a$ and $b$, as each $f_{n}$ is.

(c) For each real $\xi, K_{n}(\xi, t)$ has only real simple zeros as a function of $t$. Consider for $\xi=1-a^{2} \eta_{n}$, such a real zero $\alpha_{n}$. This leads to zeros of $f_{n}(a, t)$ located at

$$
\pm \sqrt{\frac{1-\alpha_{n}}{\eta_{n}}}
$$

If $\alpha_{n} \leq 1$, these zeros are real. However, if $\alpha_{n}>1$, we obtain simple zeros of $f_{n}$ along the imaginary axis. Since $\eta_{n} \rightarrow 0$ as $n \rightarrow \infty$, Hurwitz's Theorem shows that only zeros $\alpha_{n}$ with

$$
\alpha_{n}-1=O\left(\eta_{n}\right)
$$

lead to zeros of $f(a, t)$. We claim there is at most one such $\alpha_{n}$, leading to at most one such conjugate pair of zeros of $f(a, t)$ along the imaginary axis. To establish the claim, it suffices to show that $K_{n}(\xi, t)$ has at most one zero in $(1,1+\varepsilon)$, which lies outside $\operatorname{supp}[\mu]$. This follows from the orthogonality relation

$$
\int K_{n}(\xi, t)(\xi-t) P(t) d \mu(t)=0, \operatorname{deg}(P) \leq n-2 .
$$

If $K_{n}(\xi, t)$ has two zeros $\beta$ and $\gamma$ in $(1,1+\varepsilon)$, let

$$
P(t)=\frac{K_{n}(\xi, t)(\xi-t)}{(t-\beta)(t-\gamma)}
$$

We obtain the contradiction

$$
0=\int \frac{\left[K_{n}(\xi, t)(\xi-t)\right]^{2}}{(t-\beta)(t-\gamma)} d \mu(t)>0
$$

as $(t-\beta)(t-\gamma)$ is positive in $\operatorname{supp}[\mu]$.

\section{Lemma 4.2}

(a) Let $B>0$. Uniformly for $a \in \mathbb{R}$,

$$
f(a, a) \sim(1+|a|)^{-(2 \alpha+1)} .
$$

(b) For all $a \in \mathbb{C}$,

$$
\int_{0}^{\infty}|f(a, y)|^{2} y^{2 \alpha+1} d y \leq \frac{f(a, \bar{a})}{2 \mathbb{J}_{\alpha}^{*}(0,0)} .
$$

\section{Proof}


(a) By Lemma 3.2, for some $B>0$, and uniformly for $\frac{a^{2}}{n^{2}} \in[0, B]$, we have

$$
C_{1} \leq f_{n}(a, a)\left(a^{2}+1\right)^{\alpha+\frac{1}{2}}=\frac{K_{n}\left(1-a^{2} \eta_{n}, 1-a^{2} \eta_{n}\right)}{K_{n}(1,1)}\left(a^{2}+1\right)^{\alpha+\frac{1}{2}} \leq C_{2} .
$$

Letting $n \rightarrow \infty$ through $\mathcal{S}$, we obtain for the same $C_{1}$ and $C_{2}$, and for all $a \in[0, \infty)$,

$$
C_{1} \leq f(a, a)\left(a^{2}+1\right)^{\alpha+\frac{1}{2}} \leq C_{2} .
$$

(b) Let $r>0$. We have for all $s \in \mathbb{C}$,

$$
\begin{aligned}
K(s, \bar{s}) & =\int|K(s, t)|^{2} d \mu(t) \\
& \geq \int_{1-r^{2} \eta_{n}}^{1}|K(s, t)|^{2} \mu^{\prime}(t) d t
\end{aligned}
$$

provided $n$ is large enough. Now we make the substitutions

$$
s=1-a^{2} \eta_{n} \text { and } t=1-y^{2} \eta_{n} .
$$

For large enough $n$, we obtain

$$
\begin{aligned}
1 & \geq \int_{0}^{r}\left|\frac{K_{n}\left(1-a^{2} \eta_{n}, 1-y^{2} \eta_{n}\right)}{K_{n}(1,1)}\right|^{2}\left|\frac{K_{n}(1,1)}{K_{n}\left(1-a^{2} \eta_{n}, 1-\bar{a}^{2} \eta_{n}\right)}\right| \mu^{\prime}\left(1-y^{2} \eta_{n}\right) K_{n}(1,1) 2 y \eta_{n} d y \\
& =\int_{0}^{r} \frac{\left|f_{n}(a, y)\right|^{2}}{f_{n}(a, \bar{a})} \mu^{\prime}\left(1-y^{2} \eta_{n}\right) K_{n}(1,1) 2 y \eta_{n} d y \\
& =\int_{0}^{r} \frac{\left|f_{n}(a, y)\right|^{2}}{f_{n}(a, \bar{a})} 2 y^{2 \alpha+1} K_{n}(1,1) \eta_{n}^{\alpha+1}(1+o(1)) d y \\
& =\int_{0}^{r} \frac{|f(a, y)|^{2}}{f(a, \bar{a})} 2 y^{2 \alpha+1} \mathbb{J}_{\alpha}^{*}(0,0)(1+o(1)) d y
\end{aligned}
$$

as $n \rightarrow \infty$ through $\mathcal{S}$, recall (2.8) and (2.9). Letting $r \rightarrow \infty$ gives (4.4). We are also assuming $f(a, \bar{a}) \neq 0$. That this cannot happen follows from (4.4) and a continuity argument.

\section{Lemma 4.3}

(a) For $a \in \mathbb{R}$, let $n(f(a, \cdot), r)$ denote the the number of zeros of $f(a, \cdot)$ in the ball center 0 , radius $r$, counting multiplicity. Then for any real $a$, we have as $r \rightarrow \infty$,

$$
n(f(a, \cdot), r)-n(f(0, \cdot), r)=O(1) .
$$

(b) If $\sigma_{a}$ is the exponential type of $f(a, \cdot)$, then $\sigma_{a}$ is independent of a. 


\section{Proof}

(a) We use a basic property of

$\psi_{n}(\xi, t)=\left(\frac{\gamma_{n-1}}{\gamma_{n}}\right)^{-1} K_{n}(\xi, t)(\xi-t)=p_{n}(\xi) p_{n-1}(t)-p_{n}(t) p_{n-1}(\xi)$.

For real $\xi$, with $p_{n-1}(\xi) p_{n}(\xi) \neq 0, \psi_{n}(\xi, t)$ has, as a function of $t$, simple zeros in each of the intervals

$$
\left(x_{n n}, x_{n-1, n}\right),\left(x_{n-1, n}, x_{n-2, n}\right), \ldots,\left(x_{2 n}, x_{1 n}\right) .
$$

There is a single remaining zero, and this lies outside $\left[x_{n n}, x_{1 n}\right]$. When $p_{n-1}(\xi) p_{n}(\xi)=0, \psi_{n}(\xi, t)$ is a multiple of $p_{n}$ or $p_{n-1}$. As the zeros of the latter polynomials interlace, we see that in this case, there is a simple zero in each of the intervals

$$
\left[x_{n n}, x_{n-1, n}\right),\left[x_{n-1, n}, x_{n-2, n}\right), \ldots,\left[x_{2 n}, x_{1 n}\right) .
$$

For all this, see [5, proof of Theorem 3.1, p. 19]. It follows that whatever is $\xi$, the number $j$ of zeros of $K_{n}(\xi, t)$ in $\left[x_{m n}, x_{k n}\right]$ satisfies

$$
|j-(m-k)| \leq 1 \text {. }
$$

Consider now

$$
f_{n}(a, t)=K_{n}\left(1-a^{2} \eta_{n}, 1-t^{2} \eta_{n}\right) / K_{n}(1,1)
$$

and

$$
f_{n}(0, t)=K_{n}\left(1,1-t^{2} \eta_{n}\right) / K_{n}(1,1)
$$

as functions of $t$. In any fixed interval $[0, r]$, it follows that the difference between the number of zeros of these two functions is at most 2 . As $f_{n}(a, t)$ and $f(0, t)$ are even in $t$, the difference between the number of zeros in $[-r, r]$ is at most 4 . Moreover, the nonreal zeros of these functions, if any, occur in conjugate pairs, and lie on the imaginary axis, with at most one such pair not diverging to $\infty$ as $n \rightarrow \infty$. Letting $n \rightarrow \infty$ through $\mathcal{S}$, we see that (4.5) holds.

(b) The bound in Lemma 4.2 (b) shows that

$$
\int_{-\infty}^{\infty} \frac{\ln ^{+}|f(a, y)|}{1+y^{2}} d y<\infty
$$

so that $f(a, \cdot)$ belongs to the Cartwright class, recall (2.14). Moreover, $f(a, \cdot)$ is real valued along the real axis. By $(2.15)$,

$$
\frac{\sigma_{a}}{\pi}=\lim _{r \rightarrow \infty} \frac{n(f(a, \cdot), r)}{2 r} .
$$

In view of (a), this is independent of $a$.

In the sequel, we denote $\sigma_{a}$ by $\sigma$. 


\section{Lemma 4.4}

(a) We have for all $b \in \mathbb{R}$,

$$
\begin{aligned}
& \int_{0}^{\infty}\left(\frac{f(b / \sigma, s / \sigma)}{f(b / \sigma, b / \sigma)}-\frac{\mathbb{J}_{\alpha}^{*}\left(b^{2}, s^{2}\right)}{\mathbb{J}_{\alpha}^{*}\left(b^{2}, b^{2}\right)}\right)^{2} 2 s^{2 \alpha+1} d s \\
\leq & \frac{\sigma^{2 \alpha+2}}{f(b / \sigma, b / \sigma) \mathbb{J}_{\alpha}^{*}(0,0)}-\frac{1}{\mathbb{J}_{\alpha}^{*}\left(b^{2}, b^{2}\right)} .
\end{aligned}
$$

$$
\sigma^{2 \alpha+2} \geq \sup _{b \in \mathbb{R}} f\left(\frac{b}{\sigma}, \frac{b}{\sigma}\right) /\left\{\frac{\mathbb{J}_{\alpha}^{*}\left(b^{2}, b^{2}\right)}{\mathbb{J}_{\alpha}^{*}(0,0)}\right\} \geq 1 .
$$

In particular,

$$
\sigma \geq 1
$$

\section{Proof}

(a) First observe that

$$
\frac{f(b / \sigma, z / \sigma)}{f\left(\frac{b}{\sigma}, \frac{b}{\sigma}\right)}
$$

is an entire function in $z$, of exponential type $\leq 1$. Moreover, by Lemma $4.2(\mathrm{~b})$,

$$
\int_{0}^{\infty}\left|f\left(\frac{b}{\sigma}, \frac{s}{\sigma}\right)\right|^{2}\left(\frac{s}{\sigma}\right)^{2 \alpha+1} d \frac{s}{\sigma} \leq \frac{f\left(\frac{b}{\sigma}, \frac{b}{\sigma}\right)}{2 \mathbb{J}_{\alpha}^{*}(0,0)},
$$

and $f$ is even in each variable, so $|s|^{\alpha+\frac{1}{2}}\left|\frac{f(b / \sigma, s / \sigma)}{f(b / \sigma, b / \sigma)}\right| \in L^{2}(\mathbb{R})$. The left-hand side in (4.6) equals

$$
\begin{aligned}
& \frac{1}{f(b / \sigma, b / \sigma)^{2}} \int_{0}^{\infty} f(b / \sigma, s / \sigma)^{2} 2 s^{2 \alpha+1} d s-\frac{2}{f(b / \sigma, b / \sigma) \mathbb{J}_{\alpha}^{*}\left(b^{2}, b^{2}\right)} \int_{0}^{\infty} f\left(\frac{b}{\sigma}, \frac{s}{\sigma}\right) \mathbb{J}_{\alpha}^{*}\left(b^{2}, s^{2}\right) \\
& +\frac{1}{\mathbb{J}_{\alpha}^{* 2}\left(b^{2}, b^{2}\right)} \int_{0}^{\infty} \mathbb{J}_{\alpha}^{* 2}\left(b^{2}, s^{2}\right) 2 s^{2 \alpha+1} d s \\
\leq & \frac{\sigma^{2 \alpha+2}}{f(b / \sigma, b / \sigma) \mathbb{J}_{\alpha}^{*}(0,0)}-\frac{2}{\mathbb{J}_{\alpha}^{*}\left(b^{2}, b^{2}\right)}+\frac{1}{\mathbb{J}_{\alpha}^{*}\left(b^{2}, b^{2}\right)},
\end{aligned}
$$

by (4.9), by the reproducing kernel identity (see Theorem 6.1 below),

$$
\int_{0}^{\infty} f\left(\frac{b}{\sigma}, \frac{s}{\sigma}\right) \mathbb{J}_{\alpha}^{*}\left(b^{2}, s^{2}\right) 2 s^{2 \alpha+1} d s=f\left(\frac{b}{\sigma}, \frac{b}{\sigma}\right),
$$

and Lemma 6.2(b) below, which gives

$$
\int_{0}^{\infty} \mathbb{J}_{\alpha}^{* 2}\left(b^{2}, s^{2}\right) 2 s^{2 \alpha+1} d s=\mathbb{J}_{\alpha}^{*}\left(b^{2}, b^{2}\right) .
$$


(b) The left inequality in (4.7) follows as the left-hand side of (4.6) is non-negative. Since $f(0,0)=1$, the remaining inequalities also follow.

\section{Markov-Stieltjes Inequalities and the Proof of THEOREM 1.2}

Recall from Section 2, the Gauss type quadrature formula, with nodes $\left\{t_{j n}\right\}$ including the point 1 :

$$
\sum_{j} \lambda_{n}\left(t_{j n}\right) P\left(t_{j n}\right)=\int P(t) d \mu(t),
$$

for all polynomials $P$ of degree $\leq 2 n-2$. Recall that we order the nodes as

$$
\ldots<t_{2, n}<t_{1, n}<t_{0, n}=1<t_{-1 n}<\ldots
$$

and if $j \geq 0$, we write for some $\rho_{j n} \geq 0$,

$$
t_{j n}=1-\eta_{n} \rho_{j n}^{2}
$$

Lemma 5.1

(a) For each fixed $j \geq 0$, as $n \rightarrow \infty$ through $\mathcal{S}$,

$$
\rho_{j n} \rightarrow \rho_{j}
$$

where $\rho_{0}=0$ and

$$
0<\rho_{1} \leq \rho_{2} \leq \ldots
$$

For $j \geq 1, \rho_{j n}$ is a simple zero of $f_{n}(0, z)$.

(b) The function $f(0, z)$ has (possibly multiple) zeros at $\pm \rho_{j}, j \geq 0$, and no other zeros, except possibly for a single pair of conjugate zeros along the imaginary axis.

\section{Proof}

(a), (b) We know that $f_{n}(0, z)=K_{n}\left(1,1-z^{2} \eta_{n}\right) / K(1,1)$ has simple zeros at $\pm \rho_{j n}$, and possibly zeros on the imaginary axis, with at most one pair that does not diverge to $\infty$ as $n \rightarrow \infty$. Moreover as $n \rightarrow \infty$ through our subsequence, this sequence converges to $f(0, z)$, uniformly for $z$ in compact sets, and $f(0, z)$ is not identically 0 , since $f(0,0)=1$. The result then follows by Hurwitz' theorem.

Next, we use the Markov-Stieltjes inequalities to deduce:

\section{Lemma 5.2}


(a) For each fixed $k>\ell \geq 0$,

$$
\sum_{j=\ell+1}^{k-1} \frac{1}{f\left(\rho_{j}, \rho_{j}\right)} \leq \mathbb{J}_{\alpha}^{*}(0,0) \frac{\rho_{k}^{2 \alpha+2}-\rho_{\ell}^{2 \alpha+2}}{\alpha+1} \leq \sum_{j=\ell}^{k} \frac{1}{f\left(\rho_{j}, \rho_{j}\right)} .
$$

(b) For some $C_{1}, C_{2}>0$,

$$
\begin{aligned}
& \rho_{j+1}-\rho_{j} \leq C_{1} ; \\
& \rho_{j+2}-\rho_{j} \geq C_{2} .
\end{aligned}
$$

\section{Proof}

(a) We use the Markov-Stieltjes inequalities [5, p. 33] associated with these zeros: for each $1 \leq \ell<k \leq n$,

$$
\sum_{j: t_{j}<t_{\ell n}} \lambda_{n}\left(t_{j n}\right) \leq \int_{-\infty}^{t_{\ell n}} d \mu \leq \sum_{j: t_{j} \leq t_{\ell n}} \lambda_{n}\left(t_{j n}\right)
$$

We consider this also for $k$ :

$$
\sum_{j: t_{j}<t_{k n}} \lambda_{n}\left(t_{j n}\right) \leq \int_{-\infty}^{t_{k n}} d \mu \leq \sum_{j: t_{j} \leq t_{k n}} \lambda_{n}\left(t_{j n}\right)
$$

Assume $\ell<k$. Subtracting the relevant parts of the two-sets of inequalities yields

$$
\sum_{j: t_{k n}<t_{j n}<t_{\ell n}} \lambda_{n}\left(t_{j n}\right) \leq \int_{t_{k n}}^{t_{\ell n}} d \mu(t) \leq \sum_{j: t_{k n} \leq t_{j n} \leq t_{\ell n}} \lambda_{n}\left(t_{j n}\right) .
$$

Now assume that $t_{\ell n}, t_{k n} \in\left[1-\varepsilon_{0}, 1\right]$. Then by absolute continuity of $\mu$ in $\left[1-\varepsilon_{0}, 1\right]$, and the substitution $t=1-s^{2} \eta_{n}$, we obtain after multiplying by $K_{n}(1,1)$ that

$$
\sum_{j=\ell+1}^{k-1} \frac{K_{n}(1,1)}{K_{n}\left(t_{j n}, t_{j n}\right)} \leq 2 \int_{\rho_{\ell n}}^{\rho_{k n}} \mu^{\prime}\left(1-s^{2} \eta_{n}\right) s K_{n}(1,1) \eta_{n} d s \leq \sum_{j=\ell}^{k} \frac{K_{n}(1,1)}{K_{n}\left(t_{j n}, t_{j n}\right)} .
$$

Now let $n \rightarrow \infty$ through $\mathcal{S}$. In view of (2.8), and the previous lemma, the integral in the last line converges to

$$
2 \mathbb{J}_{\alpha}^{*}(0,0) \int_{\rho_{\ell}}^{\rho_{k}} s^{2 \alpha+1} d s=\mathbb{J}_{\alpha}^{*}(0,0) \frac{\rho_{k}^{2 \alpha+2}-\rho_{\ell}^{2 \alpha+2}}{\alpha+1} .
$$


Next, for each fixed $j \geq 0$, as $n \rightarrow \infty$ through $\mathcal{S}$,

$$
\begin{aligned}
\frac{K_{n}(1,1)}{K_{n}\left(t_{j n}, t_{j n}\right)} & =\frac{1}{f_{n}\left(\rho_{j n}, \rho_{j n}\right)} \\
& \rightarrow \frac{1}{f\left(\rho_{j}, \rho_{j}\right)} .
\end{aligned}
$$

Thus for each fixed $k, \ell,(5.3)$ follows.

(b) From (a) of this lemma, and Lemma 4.2(a),

$$
\rho_{j+1}^{2 \alpha+2}-\rho_{j}^{2 \alpha+2} \leq C \max \left\{\rho_{j}^{2 \alpha+1}, \rho_{j+1}^{2 \alpha+1}\right\} .
$$

If $\rho_{j+1}>2 \rho_{j}$, this yields

$$
\rho_{j+1}^{2 \alpha+2}\left(2^{2 \alpha+2}-1\right) \leq C \max \left\{\rho_{j}^{2 \alpha+1}, \rho_{j+1}^{2 \alpha+1}\right\}
$$

which is not possible for large enough $j$, as $\rho_{j+1} \rightarrow \infty$. Thus for large enough $j, \rho_{j} \leq \rho_{j+1} \leq 2 \rho_{j}$. The Mean Value Theorem applied in the second last inequality then shows that

$$
\left(\rho_{j+1}-\rho_{j}\right) \rho_{j+1}^{2 \alpha+1} \leq C \rho_{j+1}^{2 \alpha+1}
$$

so

$$
\rho_{j+1}-\rho_{j} \leq C
$$

The proof of (5.5) is similar.

\section{Lemma 5.3}

Let

$$
\Lambda=\limsup _{x \rightarrow \infty} \frac{f(x, x)}{\mathbb{J}_{\alpha}^{*}\left(x^{2}, x^{2}\right) / \mathbb{J}_{\alpha}^{*}(0,0)} .
$$

Let $\delta \in(0,1)$, and

$$
\beta=\left\{\begin{array}{cc}
2 \alpha+2 & \text { if } 2 \alpha+1 \geq 0 \\
-2 \alpha & \text { if } 2 \alpha+1<0
\end{array} .\right.
$$

(a) There exists $L$ such that for $k>\ell \geq L$, with

$$
\rho_{k} \leq(1+\delta) \rho_{\ell}
$$

we have

$$
k-\ell-1 \leq(1+\delta)^{\beta+1} \frac{\Lambda}{\pi}\left(\rho_{k}-\rho_{\ell}\right) .
$$

$$
\limsup _{r \rightarrow \infty} \frac{n(f(0, \cdot), r)}{2 r} \leq \frac{\Lambda}{\pi} .
$$




\section{Proof}

(a) Now by Lemma 6.4 below, we can choose $L$ such that for $j \geq L$,

$$
2 \pi \mathbb{J}_{\alpha}^{*}\left(\rho_{j}^{2}, \rho_{j}^{2}\right) \leq \frac{1+\delta}{\rho_{j}^{2 \alpha+1}} .
$$

Then for $k>\ell \geq L$,

$$
\begin{aligned}
& 2 \pi \sum_{j=\ell+1}^{k-1} \frac{\mathbb{J}_{\alpha}^{*}\left(\rho_{j}^{2}, \rho_{j}^{2}\right) / \mathbb{J}_{\alpha}^{*}(0,0)}{f\left(\rho_{j}, \rho_{j}\right)} \\
\leq & \frac{1+\delta}{\mathbb{J}_{\alpha}^{*}(0,0)} \frac{1}{\min \left\{\rho_{\ell+1}^{2 \alpha+1}, \rho_{k-1}^{2 \alpha+1}\right\}} \sum_{j=\ell+1}^{k-1} \frac{1}{f\left(\rho_{j}, \rho_{j}\right)} \\
\leq & (1+\delta) \frac{\rho_{k}^{2 \alpha+2}-\rho_{\ell}^{2 \alpha+2}}{(\alpha+1) \min \left\{\rho_{\ell+1}^{2 \alpha+1}, \rho_{k-1}^{2 \alpha+1}\right\}},
\end{aligned}
$$

by (a) of the previous lemma, and the monotonicity of the function $x^{2 \alpha+1}$. Recall too that $\rho_{j}>0$ for $j \geq 1$. By the Mean Value Theorem, and the aforementioned monotonicity, we can continue this as

$$
\begin{aligned}
& \leq 2(1+\delta)\left(\rho_{k}-\rho_{\ell}\right) \frac{\max \left\{\rho_{\ell}^{2 \alpha+1}, \rho_{k}^{2 \alpha+1}\right\}}{\min \left\{\rho_{\ell}^{2 \alpha+1}, \rho_{k}^{2 \alpha+1}\right\}} \\
& \leq\left\{\begin{array}{cc}
2(1+\delta)^{2 \alpha+2}\left(\rho_{k}-\rho_{\ell}\right), & \text { if } 2 \alpha+1 \geq 0 \\
2(1+\delta)^{-2 \alpha}\left(\rho_{k}-\rho_{\ell}\right), & \text { if } 2 \alpha+1<0
\end{array},\right.
\end{aligned}
$$

in view of (5.8). In summary, with $\beta$ as at (5.7), we have shown that

$$
2 \pi \sum_{j=\ell+1}^{k-1} \frac{\mathbb{J}_{\alpha}^{*}\left(\rho_{j}^{2}, \rho_{j}^{2}\right) / \mathbb{J}_{\alpha}^{*}(0,0)}{f\left(\rho_{j}, \rho_{j}\right)} \leq 2(1+\delta)^{\beta}\left(\rho_{k}-\rho_{\ell}\right) .
$$

We may also assume $L$ is so large that

$$
x \geq \rho_{L} \Rightarrow \frac{f(x, x)}{\mathbb{J}_{\alpha}^{*}\left(x^{2}, x^{2}\right) / \mathbb{J}_{\alpha}^{*}(0,0)} \leq \Lambda(1+\delta) .
$$

Then

$$
\frac{2 \pi}{\Lambda(1+\delta)}(k-\ell-1) \leq 2(1+\delta)^{\beta}\left(\rho_{k}-\rho_{\ell}\right)
$$

and (5.9) follows.

(b) Lemma 5.2(b) shows that $f(0, \cdot)$ has at most double zeros. Moreover, because $\left\{\rho_{j n}\right\}$ are simple zeros of $f_{n}(0, \cdot)$, (Lemma 5.1(a)), $\rho_{k}$ can only be a double zero of $f(0, \cdot)$ if it is repeated in the sequence $\left\{\rho_{j}\right\}$. Then, in the interval $\left[\rho_{\ell}, \rho_{k}\right]$, the total number of zeros of $f(0, \cdot)$, namely $k-\ell+1$ or $k-\ell+2$ or $k-\ell+3$, if 0 does not belong to $[k, \ell]$, and $k-\ell$ or $k-\ell+1$ or $k-\ell+2$ if it does, is at most $(1+\delta)^{\beta+1} \frac{\Lambda}{\pi}\left(\rho_{k}-\rho_{\ell}\right)+4$. 
Let us denote by $n[a, b]$ the number of zeros of $f(0, \cdot)$ in $[a, b]$. We have shown that for $k>\ell \geq L$ with (5.8) holding, we have

$$
n\left[\rho_{\ell}, \rho_{k}\right] \leq(1+\delta)^{\beta+1} \frac{\Lambda}{\pi}\left(\rho_{k}-\rho_{\ell}\right)+4
$$

Now let $r$ be a large positive number. Because of the spacing estimate in Lemma $5.2(\mathrm{~b})$, we can choose a positive integer $k_{1}$ such that

$$
r<\rho_{k_{1}} \leq r+C
$$

where $C$ is independent of $r$. By induction on $j$, choose $\rho_{k_{j}}, j \geq 2$, such that

$$
\rho_{k_{j}} \leq(1+\delta)^{-1} \rho_{k_{j-1}}
$$

while

$$
\rho_{k_{j}+1}>(1+\delta)^{-1} \rho_{k_{j-1}} .
$$

This decreasing sequence $\left\{\rho_{k_{j}}\right\}$ will terminate at, say, $\rho_{k_{M}}$ and we may assume $1 \leq k_{M} \leq L$. Then, using (5.11),

$$
\begin{aligned}
n[0, r] & \leq n\left[0, \rho_{k_{M}}\right]+\sum_{i=2}^{M} n\left[\rho_{k_{i}}, \rho_{k_{i-1}}\right) \\
& \leq n\left[0, \rho_{L}\right]+(1+\delta)^{\beta+1} \frac{\Lambda}{\pi} \sum_{i=2}^{M}\left(\rho_{k_{i-1}}-\rho_{k_{i}}\right)+4(M-1) \\
& =n\left[0, \rho_{L}\right]+(1+\delta)^{\beta+1} \frac{\Lambda}{\pi}\left(\rho_{k_{1}}-\rho_{k_{M}}\right)+4(M-1) \\
& \leq C_{1}+(1+\delta)^{\beta+1} \frac{\Lambda}{\pi} r+4 M,
\end{aligned}
$$

where $C_{1}=n\left[0, \rho_{L}\right]+(1+\delta)^{\beta+1} \frac{\Lambda}{\pi} C$ is independent of $r$. We claim that

$$
M \leq C \log r .
$$

Indeed, the spacing in Lemma 5.2(b) shows that

$$
n[0, r] \geq C r
$$

and moreover, necessarily by (5.12),

$$
(1+\delta)^{M-1} \rho_{1} \leq(1+\delta)^{M-1} \rho_{k_{M}} \leq \rho_{k_{1}} \leq r+C \leq 2 r
$$

so that (5.14) follows. Of course, the constant there depends on $\delta$. Dividing in (5.13) by $r$, and using (5.14), and letting $r \rightarrow \infty$, we obtain,

$$
\limsup _{r \rightarrow \infty} \frac{n[0, r]}{r} \leq(1+\delta)^{\beta+1} \frac{\Lambda}{\pi} .
$$


Since $f(0, \cdot)$ is even, and there are at most two non-real zeros, we obtain

$$
\limsup _{r \rightarrow \infty} \frac{n(f(0, \cdot), r)}{2 r} \leq(1+\delta)^{\beta+1} \frac{\Lambda}{\pi}
$$

As the left-hand side is independent of $\delta$, we can let $\delta \rightarrow 0+$ to deduce the result.

\section{Proof of Theorem 1.2}

From the lemma above, and the formula (2.15),

$$
\frac{\sigma}{\pi}=\lim _{r \rightarrow \infty} \frac{n(f(0, \cdot), r)}{2 r} \leq \frac{\Lambda}{\pi}
$$

That is,

$$
\sigma \leq \Lambda
$$

Our hypothesis (1.16) implies that

$$
f(a, a)=\lim _{n \rightarrow \infty, n \in \mathcal{S}} f_{n}(a, a)=\frac{\mathbb{J}_{\alpha}^{*}\left(a^{2}, a^{2}\right)}{\mathbb{J}_{\alpha}^{*}(0,0)}
$$

so

$$
\Lambda=\limsup _{x \rightarrow \infty} \frac{f(x, x)}{\mathbb{J}_{\alpha}^{*}\left(x^{2}, x^{2}\right) / \mathbb{J}_{\alpha}^{*}(0,0)} \leq 1
$$

and hence

$$
\sigma \leq 1
$$

We already know $\sigma \geq 1$ from Lemma 4.4. Thus

$$
\sigma=1 \text {. }
$$

Then from Lemma 4.4,

$$
\begin{aligned}
& \int_{0}^{\infty}\left(\frac{f(b, s)}{f(b, b)}-\frac{\mathbb{J}_{\alpha}^{*}\left(b^{2}, s^{2}\right)}{\mathbb{J}_{\alpha}^{*}\left(b^{2}, b^{2}\right)}\right)^{2} 2 s^{2 \alpha+1} d s \\
\leq & \frac{1}{f(b, b) \mathbb{J}_{\alpha}^{*}(0,0)}-\frac{1}{\mathbb{J}_{\alpha}^{*}\left(b^{2}, b^{2}\right)}=0,
\end{aligned}
$$

by (5.15) again. So

$$
\begin{aligned}
\frac{f(b, s)}{f(b, b)} & =\frac{\mathbb{J}_{\alpha}^{*}\left(b^{2}, s^{2}\right)}{\mathbb{J}_{\alpha}^{*}\left(b^{2}, b^{2}\right)} \\
\Rightarrow f(b, s) & =\frac{\mathbb{J}_{\alpha}^{*}\left(b^{2}, s^{2}\right)}{\mathbb{J}_{\alpha}^{*}(0,0)} .
\end{aligned}
$$

As the limit is independent of the subsequence $\mathcal{S}$, we obtain that uniformly for $a, b$ in compact subsets of the real line,

$$
\lim _{n \rightarrow \infty} \frac{K_{n}\left(1-a^{2} \eta_{n}, 1-b^{2} \eta_{n}\right)}{K_{n}(1,1)}=\frac{\mathbb{J}_{\alpha}^{*}\left(a^{2}, b^{2}\right)}{\mathbb{J}_{\alpha}^{*}(0,0)} .
$$


As the sequence in the left-hand side is entire and uniformly bounded for $a, b$ in compact subsets of $\mathbb{C}$, and the right-hand side is entire, the uniform convergence follows for $a, b$ in compact subsets of $\mathbb{C}$.

\section{The Bessel Reproducing Kernel}

The main result of this section is the following theorem:

\section{Theorem 6.1}

Let $\alpha>-1$. Let $f$ be entire of exponential type $\leq 1$, and even, with $x^{\alpha+\frac{1}{2}} f(x) \in L_{2}(0, \infty)$. Then for all complex $z$,

$$
f(z)=\int_{0}^{\infty} f(s) \mathbb{J}_{\alpha}^{*}\left(z^{2}, s^{2}\right) 2 s^{2 \alpha+1} d s .
$$

\section{Remarks}

(a) An alternative formulation is

$$
z^{\alpha} f(z)=\int_{0}^{\infty} f(s) \mathbb{J}_{\alpha}\left(z^{2}, s^{2}\right) 2 s^{\alpha+1} d s .
$$

(b) This reproducing kernel theorem ought to be well known, but we could not find a clearly statement of it. So we shall cobble it together from various results. This will be easier for $\alpha>-\frac{1}{2}$, since many results on Hankel transforms were formulated only for this range of $\alpha$.

(c) It is possible to deduce this from the $n$th reproducing kernel relation for the classical Jacobi weight $(1-x)^{\alpha}$, by scaling and taking suitable limits. Indeed, this was the author's original approach. Estimating the "tail" of the integrals is difficult, and requires Pollard's decomposition of the Christoffel-Darboux kernel. This approach is in some ways more direct, and draws less on the literature, but is far lengthier than the proof below.

Let us start with some background. The Hankel transform $H_{\alpha}$ of order $\alpha$ is defined by

$$
H_{\alpha}[g](x)=\int_{0}^{\infty} g(t) J_{\alpha}(x t)(x t)^{1 / 2} d t .
$$

It is known that this is a self-inverse unitary operator on $L_{2}(0, \infty)$. Thus, for $g \in L_{2}(0, \infty)$,

$$
\int_{0}^{\infty} H_{\alpha}[g]^{2}=\int_{0}^{\infty} g^{2}
$$

and

$$
f=H_{\alpha}[g] \Longleftrightarrow g=H_{\alpha}[f]
$$


For this, see [6, pp. 509-510], [10, p. 56], or [11, p. 83]. Let $\mathcal{H}$ be the image of $L_{2}[0,1]$ under $H_{\alpha}$. That is,

$$
\mathcal{H}=\left\{f(x)=\int_{0}^{1} g(t) J_{\alpha}(x t)(x t)^{1 / 2} d t: g \in L_{2}(0,1)\right\} .
$$

Because of the self-inversive nature of $H_{\alpha}$, we may also write

$$
\mathcal{H}=\left\{f \in L_{2}(0, \infty): H_{\alpha}[f]=0 \text { in }(1, \infty)\right\} .
$$

It is known [6, pp. 509-510], [11, p. 83] that $\mathcal{H}$ is a Hilbert space with reproducing kernel

$$
G(s, x)=\frac{(s x)^{1 / 2}}{x^{2}-s^{2}}\left\{x J_{\alpha+1}(x) J_{\alpha}(s)-s J_{\alpha+1}(s) J_{\alpha}(x)\right\} .
$$

That is, for $f \in \mathcal{H}$, we have

$$
f(x)=\int_{0}^{\infty} f(s) G(s, x) d x .
$$

Together with a suitable complex Paley-Wiener Theorem, this would quickly yield Theorem 6.1. The real Paley-Wiener theorems for Hankel transforms [2], [33] do not seem to help. Unfortunately, we have found the desired complex Paley-Wiener theorem stated only for $\alpha>-\frac{1}{2}$ :

\section{Proof of Theorem 6.1 for $\alpha>-\frac{1}{2}$}

Let $f$ be even, entire of exponential type $\leq 1$ with $x^{\alpha+\frac{1}{2}} f(x) \in L_{2}(0, \infty)$. By a result of Unni [34, Theorem 1, p. 512],

$$
f(z)=z^{-\alpha} \int_{0}^{1} t^{-\alpha} J_{\alpha}(z t) \phi(t) d t
$$

for some function $\phi$ with $t^{-\left(\alpha+\frac{1}{2}\right)} \phi(t) \in L_{2}(0,1)$. Setting $\psi(t)=0$ outside $(0,1)$, and

$$
\psi(t)=t^{-\left(\alpha+\frac{1}{2}\right)} \phi(t), t \in[0,1],
$$

we see that

$$
f(z)=z^{-\left(\alpha+\frac{1}{2}\right)} H_{\alpha}[\psi](z)
$$

and hence

$$
H_{\alpha}\left[x^{\alpha+\frac{1}{2}} f(x)\right](t)=\psi(t)=0, t \in(1, \infty) .
$$

Then the function $t^{\alpha+\frac{1}{2}} f(t)$ belongs to the Hilbert space $\mathcal{H}$, so satisfies the reproducing kernel property (6.3). That is,

$$
x^{\alpha+\frac{1}{2}} f(x)=\int_{0}^{\infty} s^{\alpha+\frac{1}{2}} f(s) G(s, x) d s .
$$


Now an alternative representation for $\mathbb{J}_{\alpha}$ is $[32$, p. 295 , eqn. (2.2) and ff.]

$$
\mathbb{J}_{\alpha}(u, v)=\frac{J_{\alpha+1}(\sqrt{u}) \sqrt{u} J_{\alpha}(\sqrt{v})-J_{\alpha+1}(\sqrt{v}) \sqrt{v} J_{\alpha}(\sqrt{u})}{2(u-v)} .
$$

Hence

$$
G(s, x)=2(s x)^{1 / 2} \mathbb{J}_{\alpha}\left(s^{2}, x^{2}\right)=2(s x)^{1 / 2+\alpha} \mathbb{J}_{\alpha}^{*}\left(s^{2}, x^{2}\right),
$$

so the identities (6.1) and (6.2) follow for real $x$. Analytic continuation gives it for complex $z$.

For general $\alpha>-1$, we proceed as follows:

\section{Lemma 6.2}

Let $\alpha>-1$. For $a, b \in(0, \infty)$,

(a)

$$
\int_{0}^{\infty} \mathbb{J}_{\alpha}\left(a^{2}, s^{2}\right) \mathbb{J}_{\alpha}\left(s^{2}, b^{2}\right) 2 s d s=\mathbb{J}_{\alpha}\left(a^{2}, b^{2}\right) .
$$

(b) For $a, b \in \mathbb{R}$,

$$
\int_{0}^{\infty} \mathbb{J}_{\alpha}^{*}\left(a^{2}, s^{2}\right) \mathbb{J}_{\alpha}^{*}\left(s^{2}, b^{2}\right) 2 s^{2 \alpha+1} d s=\mathbb{J}_{\alpha}^{*}\left(a^{2}, b^{2}\right) .
$$

(c) Let $\left\{j_{\alpha, k}\right\}_{k=1}^{\infty}$ denote the positive zeros of $J_{a}$. Then for $\ell, m \geq 1$,

$$
\int_{0}^{\infty} \mathbb{J}_{\alpha}^{*}\left(j_{\alpha, m}^{2}, s^{2}\right) \mathbb{J}_{\alpha}^{*}\left(j_{\alpha, \ell}^{2}, s^{2}\right) 2 s^{2 \alpha+1} d s=\delta_{\ell, m} \mathbb{J}_{\alpha}^{*}\left(j_{\alpha, m}^{2}, j_{\alpha, \ell}^{2}\right) \text {. }
$$

(d) Let $\left\{c_{k}\right\}_{k=1}^{\infty} \in \ell_{2}$. Then

$$
\int_{0}^{\infty}\left(\sum_{k=1}^{\infty} c_{k} \frac{\mathbb{J}_{\alpha}^{*}\left(j_{\alpha, k}^{2}, s^{2}\right)}{\sqrt{\mathbb{J}_{\alpha}^{*}\left(j_{\alpha, k}^{2}, j_{\alpha, k}^{2}\right)}}\right)^{2} 2 s^{2 \alpha+1} d s=\sum_{k=1}^{\infty} c_{k}^{2}
$$

\section{Proof}

(a), (b), We begin with the identity [32, p. 295, eqn. (2.2) and ff.]

$$
\mathbb{J}_{\alpha}(u, v)=\frac{1}{4} \int_{0}^{1} J_{\alpha}(\sqrt{u t}) J_{\alpha}(\sqrt{v t}) d t .
$$

Let us fix $v$, and define

$$
\begin{aligned}
g(s) & =\frac{1}{2}(s v)^{1 / 2} J_{\alpha}(v s) \chi_{[0,1]}(s) ; \\
h(u) & =u^{1 / 2} v^{1 / 2} \mathbb{J}_{\alpha}\left(u^{2}, v^{2}\right) .
\end{aligned}
$$


We can recast $(6.9)$ as

$$
h(u)=H_{\alpha}[g](u)
$$

and hence

$$
H_{\alpha}[h](s)=g(s)=0, s \in(1, \infty) .
$$

Then $h \in \mathcal{H}$, and we have the reproducing kernel relation (6.3) for $h$. On applying (6.4) and cancelling some powers of $u, v$, we obtain

$$
\mathbb{J}_{\alpha}\left(x^{2}, v^{2}\right)=\int_{0}^{\infty} \mathbb{J}_{\alpha}\left(x^{2}, s^{2}\right) \mathbb{J}_{\alpha}\left(s^{2}, v^{2}\right) 2 s d s .
$$

The relations (6.5) and (6.6) then follow.

(c) This follows from (b) and the fact that $\mathbb{J}_{\alpha}^{*}\left(j_{\alpha, m}^{2}, j_{\alpha, \ell}^{2}\right)=0$ when $\ell \neq m$, as follows easily from the definition (1.4) and (1.9) of $\mathbb{J}_{\alpha}^{*}$.

(d) For $m \geq 1$, let

$$
S_{m}(s)=\sum_{k=1}^{m} c_{k} \frac{\mathbb{J}_{\alpha}^{*}\left(j_{\alpha, k}^{2}, s^{2}\right)}{\sqrt{\mathbb{J}_{\alpha}^{*}\left(j_{\alpha, k}^{2}, j_{\alpha, k}^{2}\right)}}
$$

Because of (c), we have for each $n>m$,

$$
\int_{0}^{\infty}\left(S_{n}(s)-S_{m}(s)\right)^{2} 2 s^{2 \alpha+1} d s=\sum_{k=m+1}^{n} c_{k}^{2} .
$$

It follows that $\left\{S_{n}\right\}$ is a Cauchy sequence in $L_{2}[0, \infty)$ with weight $2 s^{2 \alpha+1}$, and so has a limit $S_{\infty}$ in this weighted $L_{2}$. Moreover, letting $n \rightarrow \infty$, we have

$$
\int_{0}^{\infty} S_{\infty}(s)^{2} 2 s^{2 \alpha+1} d s=\lim _{n \rightarrow \infty} \int_{0}^{\infty} S_{n}(s)^{2} 2 s^{2 \alpha+1} d s=\sum_{k=1}^{\infty} c_{k}^{2} .
$$

\section{Lemma 6.3}

Let $g$ be even and entire of exponential type $\leq 1$, with $|x|^{\alpha+1 / 2} g(x) \in$ $L_{2}(\mathbb{R})$. Then

$$
g(z)=\sum_{k=1}^{\infty} g\left(j_{\alpha, k}\right) \frac{\mathbb{J}_{\alpha}^{*}\left(j_{\alpha, k}^{2}, z^{2}\right)}{\mathbb{J}_{\alpha}^{*}\left(j_{\alpha, k}^{2}, j_{\alpha, k}^{2}\right)} .
$$

The series converge uniformly on compact sets.

\section{Proof}


We let $j_{\alpha,-k}=-j_{\alpha, k}$ denote the $k$ th negative zero of $J_{\alpha}^{*}$, for $k \geq 1$. By [9, Lemma 13, p. 57] (the notation there is defined on p. 48),

$$
g(z)=\sum_{k=-\infty, k \neq 0}^{\infty} \frac{J_{a}^{*}(z)}{J_{\alpha}^{* \prime}\left(j_{\alpha, k}\right)\left(z-j_{\alpha, k}\right)} g\left(j_{\alpha, k}\right) .
$$

Note that there $J_{\alpha}^{*}$ is denoted by $G_{\alpha}$. The uniform convergence is not stated in Lemma 13 there, but contained in the proof. Since $j_{\alpha,-k}=$ $-j_{\alpha, k}$, while $J_{\alpha}^{*}$ is even, and $J_{\alpha}^{* \prime}$ is odd, we see that

$$
\begin{aligned}
& g\left(j_{\alpha, k}\right) \frac{J_{a}^{*}(z)}{J_{\alpha}^{* \prime}\left(j_{\alpha, k}\right)\left(z-j_{\alpha, k}\right)}+g\left(j_{\alpha,-k}\right) \frac{J_{a}^{*}(z)}{J_{\alpha}^{* \prime}\left(j_{\alpha,-k}\right)\left(z-j_{\alpha,-k}\right)} \\
= & g\left(j_{\alpha, k}\right) \frac{J_{a}^{*}(z) 2 j_{\alpha, k}}{J_{\alpha}^{* \prime}\left(j_{\alpha, k}\right)\left(z^{2}-j_{\alpha, k}^{2}\right)} .
\end{aligned}
$$

Hence,

$$
g(z)=\sum_{k=1}^{\infty} g\left(j_{\alpha, k}\right) \frac{J_{a}^{*}(z) 2 j_{\alpha, k}}{J_{\alpha}^{* \prime}\left(j_{\alpha, k}\right)\left(z^{2}-j_{\alpha, k}^{2}\right)} .
$$

From (1.4) and (1.9),

$$
\mathbb{J}_{\alpha}^{*}\left(j_{\alpha, k}^{2}, z^{2}\right)=\frac{-J_{\alpha}(z) j_{\alpha, k} J_{\alpha}^{\prime}\left(j_{\alpha, k}\right)}{2\left(j_{\alpha, k}^{2}-z^{2}\right) j_{\alpha, k}^{\alpha} z^{\alpha}}=\frac{J_{\alpha}^{*}(z) j_{\alpha, k} J_{\alpha}^{* \prime}\left(j_{\alpha, k}\right)}{2\left(z^{2}-j_{\alpha, k}^{2}\right)} .
$$

Also, using (1.5) and the identities [23, p. 59, (9.16)]

$$
\begin{aligned}
x J_{\alpha}^{\prime}(x) & = \pm \alpha J_{\alpha}(x) \mp x J_{\alpha \pm 1}(x) \\
\mathbb{J}_{\alpha}^{*}\left(j_{\alpha, k}^{2}, j_{\alpha, k}^{2}\right) & =j_{\alpha, k}^{-2 \alpha} \mathbb{J}_{\alpha}\left(j_{\alpha, k}^{2}, j_{\alpha, k}^{2}\right) \\
& =\frac{j_{\alpha, k}^{-2 \alpha}}{4}\left\{-J_{\alpha+1}\left(j_{\alpha k}\right) J_{\alpha-1}\left(j_{\alpha, k}\right)\right\} \\
& =\frac{j_{\alpha, k}^{-2 \alpha}}{4} J_{a}^{\prime}\left(j_{\alpha, k}\right)^{2}=\frac{1}{4} J_{\alpha}^{* \prime}\left(j_{\alpha, k}\right)^{2} .
\end{aligned}
$$

So

$$
\frac{\mathbb{J}_{\alpha}^{*}\left(j_{\alpha, k}^{2}, z^{2}\right)}{\mathbb{J}_{\alpha}^{*}\left(j_{\alpha, k}^{2}, j_{\alpha, k}^{2}\right)}=\frac{J_{a}^{*}(z) 2 j_{\alpha, k}}{J_{\alpha}^{* \prime}\left(j_{\alpha, k}\right)\left(z^{2}-j_{\alpha, k}^{2}\right)} .
$$

Now (6.11) gives the result.

Proof of Theorem 6.1 for any $\alpha>-1$

For $m \geq 1$, let

$$
g_{m}(s)=\sum_{k=1}^{m} g\left(j_{\alpha, k}\right) \frac{\mathbb{J}_{\alpha}^{*}\left(j_{\alpha, k}^{2}, s^{2}\right)}{\mathbb{J}_{\alpha}^{*}\left(j_{\alpha, k}^{2}, j_{\alpha, k}^{2}\right)}
$$


In view of Lemma 6.2,

$$
\begin{aligned}
& \int_{0}^{\infty} \mathbb{J}_{\alpha}^{*}\left(a^{2}, s^{2}\right) g_{m}(s) 2 s^{2 \alpha+1} d s \\
= & \sum_{k=1}^{m} \frac{g\left(j_{\alpha, k}\right)}{\mathbb{J}_{\alpha}^{*}\left(j_{\alpha, k}^{2}, j_{\alpha, k}^{2}\right)} \mathbb{J}_{\alpha}^{*}\left(j_{\alpha, k}^{2}, a^{2}\right)=g_{m}(a) .
\end{aligned}
$$

Moreover, as $m \rightarrow \infty$, we have $g_{m}(a) \rightarrow g(a)$ by the previous lemma. We want to let $m \rightarrow \infty$ in the integral in the last left-hand side also. First, we note that

$$
\frac{1}{2} \sum_{k=1}^{\infty} \frac{\left|g\left(j_{\alpha, k}\right)\right|^{2}}{\mathbb{J}_{\alpha}^{*}\left(j_{\alpha, k}^{2}, j_{\alpha, k}^{2}\right)}=\int_{0}^{\infty} x^{2 \alpha+1} g(x)^{2} d x<\infty .
$$

Indeed, since $|x|^{2 \alpha+1} g(x)^{2} \in L_{1}(\mathbb{R})$, and $g^{2}$ is of type $\leq 2$, a quadrature formula of Grozev and Rahman [4, Theorem 5.1*, p. 118], [8, Theorem 4, p. 717] asserts that

$$
\int_{0}^{\infty} x^{2 \alpha+1} g(x)^{2} d x=2 \sum_{k=1}^{\infty}{\frac{g\left(j_{\alpha, k}\right)}{\left|J_{\alpha}^{* \prime}\left(j_{\alpha, k}\right)\right|}}^{2} .
$$

Then (6.12) gives (6.14). It follows easily from (6.14) that $\left\{g_{n}\right\}_{n=1}^{\infty}$ is a Cauchy sequence in $L_{2}(\mathbb{R})$, with weight $x^{2 \alpha+1}$. Since $g_{n}(x) \rightarrow g(x)$ uniformly for $x$ in compact sets, we deduce that

$$
\lim _{n \rightarrow \infty} \int_{0}^{\infty}\left(g_{n}(x)-g(x)\right)^{2} x^{2 \alpha+1} d x=0 .
$$

Then, using (6.13),

$$
\begin{aligned}
& \left|\int_{0}^{\infty} \mathbb{J}_{\alpha}^{*}\left(a^{2}, s^{2}\right) g(s) 2 s^{2 \alpha+1} d s-g(a)\right| \\
= & \left|\int_{0}^{\infty} \mathbb{J}_{\alpha}^{*}\left(a^{2}, s^{2}\right)\left(g(s)-g_{n}(s)\right) 2 s^{2 \alpha+1} d s+g_{n}(a)-g(a)\right| \\
\leq & \left(\int_{0}^{\infty} \mathbb{J}_{\alpha}^{*}\left(a^{2}, s^{2}\right)^{2} 2 s^{2 \alpha+1} d s\right)^{1 / 2}\left(\int_{0}^{\infty}\left(g(s)-g_{n}(s)\right)^{2} 2 s^{2 \alpha+1} d s\right)^{1 / 2} \\
& +\left|g_{n}(a)-g(a)\right| \\
\rightarrow & 0, n \rightarrow \infty .
\end{aligned}
$$

Here we also used Lemma 6.2 to deduce

$$
\int_{0}^{\infty} \mathbb{J}_{\alpha}^{*}\left(a, s^{2}\right)^{2} 2 s^{2 \alpha+1} d s<\infty .
$$




\section{Lemma 6.4}

As $x \rightarrow \infty$,

$$
\mathbb{J}_{\alpha}^{*}\left(x^{2}, x^{2}\right)=\frac{1}{2 \pi x^{2 \alpha+1}}(1+o(1))
$$

\section{Proof}

$J_{\alpha}$ admits the following asymptotic [1, p. 364, (9.2.1)]: as $x \rightarrow \infty$,

$$
J_{\alpha}(x)=\sqrt{\frac{2}{\pi x}}\left[\cos \left(x-\frac{\alpha \pi}{2}-\frac{\pi}{4}\right)+O\left(x^{-1}\right)\right] .
$$

For large $x$, let us write

$$
t=t(x)=\sqrt{x}-\frac{\alpha \pi}{2}-\frac{\pi}{4} .
$$

Then

so as $x \rightarrow \infty$,

$$
\begin{gathered}
J_{\alpha}(\sqrt{x})=\sqrt{\frac{2}{\pi \sqrt{x}}}\left[\cos t+O\left(x^{-1 / 2}\right)\right] ; \\
J_{\alpha+1}(\sqrt{x})=\sqrt{\frac{2}{\pi \sqrt{x}}\left[\sin t+O\left(x^{-1 / 2}\right)\right] ;} \\
J_{\alpha-1}(\sqrt{x})=\sqrt{\frac{2}{\pi \sqrt{x}}}\left[-\sin t+O\left(x^{-1 / 2}\right)\right] ;
\end{gathered}
$$

$\mathbb{J}_{\alpha}(x, x)=\frac{1}{4}\left\{J_{\alpha}(\sqrt{x})^{2}-J_{\alpha+1}(\sqrt{x}) J_{\alpha-1}(\sqrt{x})\right\}=\frac{1}{2 \pi \sqrt{x}}(1+o(1))$.

Finally (6.15) follows from the definition of $\mathbb{d}_{\alpha}^{*}$.

\section{REFERENCES}

[1] M. Abramowitz and I. Stegun, Handbook of Mathematical Functions, Dover, New York, 1965.

[2] N.B. Andersen, Real Paley-Wiener Theorems for the Hankel Transform, J. Fourier Anal, Applns., 12(2006), 17-25.

[3] P. Deift, Orthogonal Polynomials and Random Matrices: A Riemann-Hilbert Approach, Courant Institute Lecture Notes, Vol. 3, New York University Pres, New York, 1999.

[4] D.P. Dryanov, M.A. Qazi, Q. I. Rahman, Entire Functions of Exponential Type in Approximation Theory, (in) Constructive Theory of Functions, Varna 2002, (ed. B. Bojaov), DARBA, Sofia, 2003, pp. 86-135.

[5] G. Freud, Orthogonal Polynomials, Pergamon Press/ Akademiai Kiado, Budapest, 1971.

[6] A.G. Garcia, Orthogonal Sampling Formulas: A Unified Approach, SIAM Review, 42(2000), 499-512.

[7] I.S. Gradshteyn and I.M. Ryzhik, Table of Integrals, Series and Products, Academic Press, San Diego, 1980. 
[8] G.R. Grozev, Q.I. Rahman, A Quadrature Formula involving zeros of Bessel Functions as nodes, Math. Comp., 64(1995), 715-725.

[9] G.R. Grozev, Q.I. Rahman, Lagrange Interpolation in the Zeros of Bessel functions by Entire Functions of Exponential Type and Mean Convergence, Methods and Applications of Analysis, 3(1996), 46-79.

[10] J.R. Higgins, Completeness and Basis Properties of Sets of Special Functions, Cambridge University Press, Cambridge, 1977.

[11] J.R. Higgins, Sampling Theory in Fourier and Signal Analysis: Foundations, Oxford University Press, Oxford, 1996.

[12] K.G. Ivanov and V. Totik, Fast Decreasing Polynomials, Constr. Approx., 6(1990), 1-20.

[13] P. Koosis, The Logarithmic Integral I, Cambridge University Press, Cambridge, 1988.

[14] A.B. Kuijlaars and M. Vanlessen, Universality for Eigenvalue Correlations from the Modified Jacobi Unitary Ensemble, International Maths. Research Notices, 30(2002), 1575-1600.

[15] B. Ya. Levin, in collaboration with Yu. Lyubarskii, M. Sodin, V. Tkachenko, Lectures on Entire Functions, Translations of Mathematical Monographs, Vol. 150, American Mathematical Society, Providence, 1996.

[16] Eli Levin and D.S. Lubinsky, Universality Limits Involving Orthogonal Polynomials on the Unit Circle, Computational Methods and Function Theory, $7(2007), 543-561$.

[17] Eli Levin and D.S. Lubinsky, Universality Limits in the Bulk for Varying Measures, manuscript.

[18] D.S. Lubinsky, A New Approach to Universality Limits involving Orthogonal Polynomials, to appear in Annals of Mathematics.

[19] D.S. Lubinsky, A New Approach to Universality Limits at the Edge of the Spectrum, to appear in Contemporary Mathematics.

[20] D.S. Lubinsky, Universality Limits in the Bulk for Arbitrary Measures on Compact Sets, to appear in J. d'Analyse de Math.

[21] P. Nevai, Orthogonal Polynomials, Memoirs of the AMS no. 213 (1979).

[22] P. Nevai, Geza Freud, Orthogonal Polynomials and Christoffel Functions: A Case Study, J. Approx. Theory, 48(1986), 3-167.

[23] F.W.J. Olver, Asymptotics and Special Functions, Academic Press, San Diego, 1974.

[24] T. Ransford, Potential Theory in the Complex Plane, Cambridge University Press, Cambridge, 1995.

[25] E.B. Saff and V. Totik, Logarithmic Potentials with External Fields, Springer, New York, 1997.

[26] B. Simon, Orthogonal Polynomials on the Unit Circle, Parts 1 and 2, American Mathematical Society, Providence, 2005.

[27] B. Simon, Two Extensions of Lubinsky's Universality Theorem, to appear in J. d'Analyse de Math.

[28] H. Stahl and V. Totik, General Orthogonal Polynomials, Cambridge University Press, Cambridge, 1992.

[29] G. Szegö, Orthogonal Polynomials, American Mathematical Society Colloquium Publications, American Mathematical Society, Providence, 1975. 
[30] V. Totik, Asymptotics for Christoffel Functions for General Measures on the Real Line, J. d'Analyse Math., 81(2000), 283-303.

[31] V. Totik, Universality and fine zero spacing on general sets, manuscript.

[32] C.A. Tracy and H. Widom, Level Spacing Distributions and the Bessel Kernel, Commun. Math. Phys., 161(1994), 289-309.

[33] V.K. Tuan, On the Range of the Hankel and Extended Hankel Transforms, J. Math. Anal. Applns., 209(1997), 460-478.

[34] K.R. Unni, Hankel Transforms and Entire Functions, Bull. Amer. Math. Soc., 71(1965), 511-513.

School of Mathematics, Georgia Institute of Technology, Atlanta, GA 30332-0160, USA., LUBINSKY@MATH.GATECH.EDU 